\title{
¿Cuál es el nivel de contaminación del cepillo de dientes almacenado en diferentes entornos sanitarios?
}

\section{What is the level of toothbrush contamination stored in different sanitary settings?}

Medina-Patruno $C^{*}$, Bolaños-Rivero $M^{* *}$, Martín-Sánchez $A M^{* * *}$, Saavedra-Santana $\mathrm{P}^{* * * *}$, Vicente-Barrero $M^{* * * * *}$

\section{RESUMEN}

Introducción. Los cepillos pueden albergar una amplia variedad de microorganismos, incluyendo bacterias hongos y virus, pudiendo así facilitar la translocación de especies en un mismo individuo y la transmisión de especies entre individuos.

Metodos. Estudio de la contaminación bacteriana de los cepillos de dientes de cien voluntarios con la cumplimentación de un cuestionario (lugar de almacenamiento, tipo de cepillo, utilización de estuche de almacenamiento y meses de uso).

Resultados. Se estudiaron un total de 100 muestras. El 33\% guardaba el cepillo fuera del baño, un $20 \%$ lo guardaba en el cajón del baño y un $47 \%$ sobre el lavabo del baño.

El 92\% de las muestras estaban contaminadas como mínimo con un grupo de microorganismos

Conclusión. El lugar de almacenamiento del cepillo de dientes es fundamental en la reducción de la contaminación. Se debe guardar en un lugar seco, lejos del inodoro y sin estuche.

PALABRAS CLAVE: Contaminación microbiana; higiene oral, contaminación de los cepillos dentales.

\begin{abstract}
Introduction. Brushes can house a wide variety of microorganisms, including fungal bacteria and viruses, which can facilitate the translocation of species in the same individual and the transmission of species between individuals. Methods. Study of the bacterial contamination of toothbrushes of one hundred volunteers with the completion of a questionnaire (place of storage, type of brush, use of storage case and months of use).

Results. A total of 100 samples were studied. 33\% kept the brush outside the bathroom, $20 \%$ kept it in the bathroom drawer and $47 \%$ on the bathroom sink.

$92 \%$ of the samples were contaminated with at least one group of microorganisms.

Conclusion. The storage place of the toothbrush is fundamental in the reduction of contamination. It should be stored in a dry place, away from the toilet and without a box.

* Graduada en Medicina. Complejo Hospitalario Materno-Insular Las Palmas de Gran Canaria.

* * Doctora en Medicina. Especialista en Microbiología. Complejo Hospitalario Materno-Insular Las Palmas de Gran Canaria.

*** Catedrático de Microbiología. Universidad de Las Palmas de Gran Canaria.

**** Catedrático de Estadística e Investigación Operativa. Universidad de Las Palmas de Gran Canaria.

$* * * *$ Doctor en Medicina. Especialista en Estomatología. Complejo Hospitalario Materno-Insular Las Palmas de Gran Canaria. Profesor Asociado a la Universidad de Las Palmas de Gran Canaria.
\end{abstract}


KEY WORDS: Microbial contamination; oral hygiene; Toothbrush contamination.

Fecha de recepción: 18 de febrero de 2019.

Fecha de aceptación: 9 de marzo de 2019.

Medina-Patruno C, Bolaños-Rivero M, Martín-Sánchez AM, Saavedra-Santana P, Vicente-Barrero M.

¿Cuál es el nivel de contaminación del cepillo de dientes almacenado en diferentes entornos sanitarios?

2019; 35, (2): 69-72

\section{INTRODUCCIÓN}

La cavidad bucal contiene diferentes microambientes, cada uno con su propia microbiota. Sin embargo, las especies comunes a todos los lugares de la cavidad bucal pertenecen a los géneros Gemella, Granulicatella, Veillonella y Streptococcus $^{(1)}$. Esta última se ha reportado como el género preponderante a nivel de esta cavidad y se han diferenciado más de 16 especies de esta bacteria, siendo las más frecuentes Streptococcus mutans, Streptococcus intermedius, Streptococcus oralis $y$ Streptococcus sanguinis.

Entre los instrumentos empleados para la higiene bucal, el cepillo dental es el más importante para remover la placa. No obstante, no existe un consenso en cuanto al tiempo de uso y lugar de almacenamiento por lo que las cerdas pueden llegar a ser un medio idóneo para la proliferación y desarrollo de microorganismos provenientes tanto del medio ambiente como de la propia cavidad bucal ${ }^{(2,3)}$.

Nuestro objetivo en este estudio es identificar los factores asociados con la contaminación del cepillo de dientes por gérmenes, estableciendo una relación entre la forma de guardar el cepillo de dientes, en un lugar determinado y el tiempo de uso, con el tipo de microorganismos aislados en ellos. Intentamos diferenciar además si la posible contaminación es microbiota bucal o bacilos gram negativos.

\section{MATERIAL Y MÉTODOS}

Presentamos un estudio transversal, del cultivo e identificación de microorganismos en cepillos de dientes de 100 voluntarios, estudiantes de Medicina, a los que se les entregó un cuestionario con las siguientes variables: edad, sexo, tipo de ce- pillo, lugar de almacenamiento, meses de uso y utilización de estuche para el cepillo.

Se cortaron varias cerdas de cada cepillo con un bisturí estéril. Las cerdas fueron guardadas individualmente en un contenedor estéril y transportadas en el intervalo de una hora al Servicio de Microbiología. Posteriormente se suspendieron las cerdas en un medio de cultivo líquido (tioglicolato) e incubados en la estufa durante 18 horas a $35^{\circ} \mathrm{C}$. Estos tioglicolatos fueron sembrados en medio sólido (agar sangre, chocolate y Mc Conkey) y se incubaron de nuevo en estufa durante 18 horas a $35^{\circ} \mathrm{C}$.

\section{AnÁlisis estadístico.}

Las variables categóricas se expresan como frecuencias y porcentajes; media y desviación estándar (DE) cuando los datos siguieron una distribución normal, o como mediana y rango intercuartílico (IQR $=25^{\circ}-75^{\circ}$ percentil) cuando la distribución se salió de la normalidad. Los porcentajes se compararon utilizando la prueba de Chi-cuadrado (x2) o la prueba exacta de Fisher. Las medias por el t-test y las medianas por la prueba de Wilcoxon para datos independientes. La significación estadística se estableció en $\mathrm{p}<.05$.

Los datos se analizaron utilizando el paquete $R$, versión 3.3.1 (R Core Team de desarrollo, 2016).

\section{RESULTADOS}

Se estudiaron un total de 100 alumnos, de los cuales un $31 \%$ eran hombres y un $69 \%$ eran mujeres. Con una media edad de 22,5 años y una deviación estándar de 1.80.

En la muestra, un $33 \%$ de pacientes guardaban el 
Tabla 1. Variables a estudiar

\begin{tabular}{lc}
\multicolumn{2}{c}{ Tabla 1. Variables a estudiar } \\
$\mathrm{N}$ & 100 \\
\hline Age, yeas & $22.5 \pm 1.8$ \\
\hline Sex Female & $69(69.0)$ \\
\hline Cap & $56(56.0)$ \\
Type of toothbrush & $12(12.0)$ \\
Electric & $88(88.0)$ \\
\hline Manual & $20(20.0)$ \\
Type of toothbrush & $47(47.0)$ \\
Cajón & $33(33.0)$ \\
\hline Lavabo & $4(2-6)$ \\
\hline Out & $61(61.0)$ \\
\hline Months of use & $44(44.0)$ \\
\hline Flora Normal & \\
\hline Bacilos gram negativo &
\end{tabular}

cepillo fuera del baño, un $20 \%$ lo guardaba en el cajón del baño y un $47 \%$ en el lavabo.

La Tabla 1 resume las características de la muestra (número de sujetos, sexo y edad), así como la de los cepillos de dientes (tipo de cepillo de dientes, tiempo de uso, uso de estuche y lugar de almacenamiento) y las prevalencias de su contaminación.

En 61 muestras encontramos microbiota habitual $\mathrm{y}$ en 44 bacilos gram negativos.

En la Tabla 2 se analizaron todas las variables. Encontramos estadísticamente significativo aislar bacilos gram negativos en aquellos cepillos que se guardan en un estuche $(p=0,003)$. Los meses de uso del cepillo sin recambiar, no fue estadísticamente significativo $(p=0,317)$.

\section{DISCUSIÓN}

En nuestro estudio, demostramos que los cepillos de dientes están ampliamente contaminados pues el $92 \%$ dio positivo como mínimo a un grupo de microorganismos.

$\mathrm{Al}$ igual que varios estudios realizados previamente, los grupos bacterianos contaminantes que han predominado en nuestros resultados han sido, Streptococcus del grupo viridans (39\%), bacilos gram negativos lactosa positivo (40\%) y Staphylococcus coagulasa negativos $(35 \%)^{(4-8)}$.

Según los estudios de Chirag ${ }^{(7)}$ y Glass ${ }^{(8)}$, los meses de uso del cepillo de dientes constituye un factor importante en la contaminación. Este demostró que los cepillos usados durante tres meses tenían una contaminación mayor que aquellos usados durante un mes. Por el contrario, en nuestro estudio se aprecia que el único microorganismo con una significación estadística y afectado por la variable meses de uso es Candida albicans. Poco representativo ya que son solo dos casos, pero curiosamente con poco tiempo de uso.

Por el contrario, nuestros resultados coinciden con los obtenidos por Abhishek ${ }^{(10)}$ y Susheela ${ }^{(11)}$, en los que el uso del estuche conduce al crecimiento de microorganismos oportunistas como bacilos gram negativos lactosa positivo. Podría tratarse de contaminación fecal, por aerosoles del inodoro. Hay que tener en cuenta que la acción de vaciar el sanitario genera aerosoles con microorganismos fecales que pueden contaminar los cepillos de dientes. Díaz-Caballero et al. ${ }^{(3)}$ en un estudio sobre la distancia máxima que alcanza la descarga del inodoro, postuló que la distancia promedio de los cepillos dentales al sanitario es de $108 \mathrm{~cm}$, mientras que la salpicadura máxima alcanzada fue de $145 \mathrm{~cm}$, lo que permite concluir, que los cepillos dentales se encuentran dentro del área de salpicadura. La conclusión que obtu-

\begin{tabular}{|c|c|c|c|c|c|c|c|}
\hline Flora normal & Muestra & Edad en años & Meses de uso & Antibióticos & Estuche & Eléctrico & Fuera del baño \\
\hline No & $\mathrm{n}=39$ & $23(22-23)$ & $3(2-5)$ & $3(7.7)$ & $24(61.5)$ & $4(10.3)$ & $16(41.0)$ \\
\hline $\mathrm{Si}$ & $N=61$ & $23(21-23)$ & $4(2-7)$ & $5(8.2)$ & $32(52.5)$ & $8(13.1)$ & $17(27.9)$ \\
\hline p-values & & $P=0.816$ & $P=0.489$ & $\mathrm{P}=1$ & $\mathrm{P}=0.372$ & $\mathrm{P}=0.761$ & $P=0.172$ \\
\hline \multicolumn{8}{|l|}{$\begin{array}{l}\text { Bacilos } \\
\text { gram negativo }\end{array}$} \\
\hline No & $\mathrm{n}=56$ & $23(21-24)$ & $4(3-7)$ & $4(7.1)$ & $24(42.9)$ & $4(7.1)$ & $14(25.0)$ \\
\hline Si & $\mathrm{n}=44$ & $22(21-23)$ & $3.5(2-5)$ & $4(9.1)$ & $32(72.7)$ & $8(18.2)$ & $19(43.2)$ \\
\hline p-values & & $P=0.124$ & $P=0.317$ & $P=0.728$ & $P=0.003$ & $\mathrm{P}=0.092$ & $P=0.055$ \\
\hline
\end{tabular}


vo Karibasappa et al. ${ }^{(5)}$ en su estudio, es que los cepillos de dientes no se deberían guardar en el baño. Algunos autores, dando por hecho que es imposible impedir la contaminación, recomiendan sumergirlos en alguna sustancia antibacteriana después de cada uso ${ }^{(5)}$. Incluso bastaría mantener en contacto el cepillo con la pasta dentífrica entre uso y uso para reducir la contaminación ${ }^{(6)}$.

Cuando se almacenan varios cepillos en un mismo recipiente, la distancia de almacenamiento entre cepillo y cepillo, es mínima $(2 \mathrm{~cm})$, lo que puede favorecer la contaminación por contacto entre ellos durante su estancia en el cepillero.

\section{CONCLUSIONES}

1. La contaminación ha predominado en nuestros resultados. El 92\% de los cepillos de dientes estaban contaminados con microorganismos.

2. Las especies contaminantes fueron Streptococcus del grupo viridans (39\%), gram-negative bacilos gram negativos lactosa positivo (40\%) y Staphylococcus coagulasa negativos (35\%).

3. El uso del estuche para guardar el cepillo entre cepillado y cepillado, genera un microambiente propicio para la proliferación de bacilos gérmenes gram negativos.

\section{BIBLIOGRAFIA}

1. Aas, J. A., Paster, B. J., Stokes, L. N., Olsen, I., and Dewhirst, F. E. Defining the normal bacterial flora of the oral cavity. Journal of Clinical Microbiology. 2005; 43: 5721 - 32.

2. American Dental Association. Statement on toothbrush care: clearing, storage and replacement. Council on Scientific Affairs, November 2011. [consultada el 28 de junio de 2018] https://www.ada.org/en/about-theada/ada-positions-policies-and-statements/ statement-on-toothbrush-care-cleaning-storage-and-

3. Díaz-Caballero AJ, León EE, Montoya ME, Vivero LE, Abello RE. Evaluation of the maxim splash area of toilet discharges and its relation with the place of the toothbrushes in ba- throoms of Cartagena, Colombia. Univ Odontol. 2002; 22 :31-6

4. Nelson-Filho P, Faria G, da Silva RA, Rossi MA, Ito IY. Evaluation of the contamination and disinfection methods of toothbrushes used by 24 - to 48-month-old children. J Dent Child. 2006; 73: 152-8.

5. Karibasappa GN, Nagesh L, Sujatha BK. Assessment of microbial contamination of toothbrush head: An in vitro study. Indian J Dent Res. 2011; 22: 2-5.

6. Efstratiou M, Papaioannou W, Nakou M, Ktenas E, Vrotsos IA, Panis V. Contamination of a toothbrush with antibacterial properties by oral microorganisms. J Dent. 2007; 35: 331-7.

7. Chirag M. R, Ruchi A., Deepak P. B., Subha D., Abhinandan A. K., Vikram S. Assessment of microbial contamination on twice a day used toothbrush head after 1 - month and 3 months: An in vitro study. J Nat Sci Biol Med. 2015; 6: 44-8.

8. Sammons RL, Kaur D, Neal P. Bacterial survival and biofilm formation on conventional and antibacterial tooth brushes. Biofilms 2004;1:123-30. https://doi.org/10.1017/ S1479050504001334.

9. Glass R, Lare M. Toothbrush contamination: a potential health risk? Quintessence Int 1988; 17:39-42.

10. Mehta A, Sequeira PS, Bhat G. Bacterial contamination and decontamination of toothbrushes after use. N Y State Dent J. 2007; 73:20-2.

11. Susheela P. and Radha R. Studies on the microbiological contamination of toothbrushes and importance of decontamination using disinfectants. World journal of pharmaceutical and medical research. 2016; 2:201-7

\section{AUTOR DE CORRESPONDENCIA:}

Mario Vicente-Barrero

c/ Alcalde Henríquez Pitti $13,1^{\circ}$ izq

35400- Arucas. Las Palmas

mmvicenteb@gmail.com 